\title{
Single-Trial Phase Entrainment of Theta Oscillations in Sensory Regions Predicts Human Associative Memory Performance
}

\author{
Danying Wang, Andrew Clouter, Qiaoyu Chen, Kimron L. Shapiro, and $₫$ Simon Hanslmayr \\ School of Psychology, Centre for Human Brain Health, University of Birmingham, Birmingham B15 2TT, United Kingdom
}

Episodic memories are rich in sensory information and often contain integrated information from different sensory modalities. For instance, we can store memories of a recent concert with visual and auditory impressions being integrated in one episode. Theta oscillations have recently been implicated in playing a causal role synchronizing and effectively binding the different modalities together in memory. However, an open question is whether momentary fluctuations in theta synchronization predict the likelihood of associative memory formation for multisensory events. To address this question we entrained the visual and auditory cortex at theta frequency $(4 \mathrm{~Hz})$ and in a synchronous or asynchronous manner by modulating the luminance and volume of movies and sounds at $4 \mathrm{~Hz}$, with a phase offset at $0^{\circ}$ or $180^{\circ}$. EEG activity from human subjects (both sexes) was recorded while they memorized the association between a movie and a sound. Associative memory performance was significantly enhanced in the $0^{\circ}$ compared with the $180^{\circ}$ condition. Source-level analysis demonstrated that the physical stimuli effectively entrained their respective cortical areas with a corresponding phase offset. The findings suggested a successful replication of a previous study (Clouter et al., 2017). Importantly, the strength of entrainment during encoding correlated with the efficacy of associative memory such that small phase differences between visual and auditory cortex predicted a high likelihood of correct retrieval in a later recall test. These findings suggest that theta oscillations serve a specific function in the episodic memory system: binding the contents of different modalities into coherent memory episodes.

Key words: binding; hippocampus; memory; oscillations; STDP; theta

\section{Significance Statement}

How multisensory experiences are bound to form a coherent episodic memory representation is one of the fundamental questions in human episodic memory research. Evidence from animal literature suggests that the relative timing between an input and theta oscillations in the hippocampus is crucial for memory formation. We precisely controlled the timing between visual and auditory stimuli and the neural oscillations at $4 \mathrm{~Hz}$ using a multisensory entrainment paradigm. Human associative memory formation depends on coincident timing between sensory streams processed by the corresponding brain regions. We provide evidence for a significant role of relative timing of neural theta activity in human episodic memory on a single-trial level, which reveals a crucial mechanism underlying human episodic memory.

\section{Introduction}

A week after seeing your favorite band in concert, while sitting in your office, you mentally travel back and re-experience the

Received Feb. 7, 2018; revised May 22, 2018; accepted May 24, 2018.

Author contributions:D.W. and S.H. wrote the first draft of the paper; D.W., A.C., K.L.S., and S.H. edited the paper; D.W., A.C., K.L.S., and S.H. designed research; D.W. and Q.C. performed research; A.C. contributed unpublished reagents/analytic tools; D.W. analyzed data; D.W. and S.H. wrote the paper.

This work was supported by the ERC Grant Code4Memory (647954) awarded to S.H., who is further supported by the Wolfson Society and the Royal Society.

The authors declare no competing financial interests.

Correspondence should be addressed to Dr. Simon Hanslmayr, School of Psychology, Centre for Human Brain Health, University of Birmingham, Birmingham B15 2TT, UK. E-mail: s.hansImayr@bham.ac.uk.

DOI:10.1523/JNEUROSCI.0349-18.2018

Copyright $\odot 2018$ the authors $\quad 0270-6474 / 18 / 386299-11 \$ 15.00 / 0$ band playing your favorite song. Episodic memory is the machinery that allows us to form, hold, and revisit such rich, often multisensory, memories (Tulving, 2002). Yet sensory information is processed in specialized brain regions distributed across the cortex, which raises the fundamental question of how the different sensory elements are bound into a coherent memory representation and, more importantly, what affects the likelihood of that association being made. In this study, we address this question using a recently developed multisensory entrainment paradigm (Clouter et al., 2017). We show that memory formation for dynamic audiovisual events can be predicted by momentary fluctuations in theta phase synchronization between sensory regions. 
The hippocampus plays a fundamental role in episodic memory formation (Scoville and Milner, 1957) and receives highly preprocessed information from virtually all sensory modalities (Moscovitch, 2008; Muzzio et al., 2009). This makes it a likely region supporting the binding function in memory. Importantly, within the hippocampus synapses are more likely to undergo modification if the inputs are active at the same time (Bliss and Collingridge, 1993). Theta oscillations, a dominant signal in the hippocampus (Jacobs, 2014), synchronize neural ensembles (Buzsáki, 2010) and thus likely establish the synchrony required to effectively induce synaptic plasticity. Studies in rodents suggest that synaptic plasticity depends on the phase of the ongoing theta rhythm. Stimulation in the hippocampus yields either long-term potentiation (LTP) or long-term depression, depending on whether stimulation is applied at the peak or trough of the theta phase, respectively (Pavlides et al., 1988; Huerta and Lisman, 1995; Hölscher et al., 1997; Hyman et al., 2003).This theta-phasedependent learning mechanism has sparked considerable interest in human memory research and is an important component of several computational models of human memory (Hasselmo, 2005; Norman et al., 2006; Parish et al., 2018). However, direct evidence for such a theta-dependent synchronization mechanism in human memory is scarce. Such direct evidence would have to show two things: (1) externally synchronizing/desynchronizing sensory regions in theta frequency impacts memory formation and (2) momentary fluctuations in interareal phase synchronization predict memory formation. By using a multisensory entrainment paradigm, Clouter et al. (2017) successfully provided evidence on the first hypothesis. Auditory and visual brain regions were entrained at theta frequency $(4 \mathrm{~Hz})$ and at intended phase offsets $\left(0,90,180\right.$, and $\left.270^{\circ}\right)$ by amplitude/luminance modulated multisensory stimuli. Clouter et al. (2017) showed that such neural entrainment could impact associative memory performance on an averaged trials level, such that trials from the $0^{\circ}$ phase offset condition were significantly better memorized compared with the other phase offset conditions $(90,180$, and $270^{\circ}$ ). Importantly, such memory enhancement was only shown when the stimuli were modulated at $4 \mathrm{~Hz}$ but not at other frequency bands $(1.7$ or $10.5 \mathrm{~Hz})$.

A crucial question, which the previous study was unable to answer is whether on a single-trial level the strength of entrainment would be related to the memory outcome. Specifically, can the variance in single-trial synchronization explain to some extent the variance in memory performance? Such a finding would provide a more direct link between theta phase synchronization and associative memory formation, and would support the causal role of relative timing between cortical inputs and theta oscillations for human episodic memory formation. To address this question we here used the same paradigm as by Clouter et al. (2017) while recording participants' electrical brain activity using 128 channel EEG.

\section{Materials and Methods}

Participants. Thirty-one healthy English-speaking young adults participated in the experiment (15 male; mean age: 21.48 years; range: $18-29$ years), which was an entirely new group of participants who did not take part in any experiment reported by Clouter et al. (2017). All participants had normal or corrected-to-normal vision and normal hearing. One participant was ambidextrous; all other participants were right-handed. Four participants were given course credits via the University of Birmingham's Psychology Research Participation Scheme. The remaining 27 participants were paid $£ 6$ per hour for their participation. The data from three participants were excluded because of their poor, chance-level memory performance. The data from one participant was excluded due to a technical problem during EEG recording. The data from another three participants were excluded due to poor EEG data quality. The data from the remaining 24 participants were retained for the final data analysis.

Stimulus material. The visual and auditory stimuli used were taken from the same pool as those used in by Clouter et al. (2017), their Experiment 3. Movie clips $(N=192)$ were presented for $3 \mathrm{~s}$ in total 76 frames at a frame rate of 25 frames/s. The luminance of the movie clips was modulated from 0 to $100 \%$ with at $4 \mathrm{~Hz}$ with sine wave, with all movie clips initially starting at $50 \%$ luminance (Fig. 1). On each trial, concurrent with the presentation of the movie, 1 of 192 sound clips was presented for $3 \mathrm{~s}$, with a lag of $40 \mathrm{~ms}$ (i.e., starting $40 \mathrm{~ms}$ after the start of the movie). This $40 \mathrm{~ms}$ lag was necessary to account for the fact that auditory stimuli are processed faster than visual stimuli, and therefore to ensure a lag at the targeted phase difference of 0 and 180 between auditory and visual cortex (Clouter et al., 2017). The amplitude of the sound clips was modulated from 0 to $100 \%$ at $4 \mathrm{~Hz}$ with a sine wave, with all sound clips starting at $0 \%$ amplitude, and ramping in linearly. Each sound was modulated both at 0 and $180^{\circ}$ phase offsets from sine-wave-modulated movies (accounting for the $40 \mathrm{~ms}$ lag for the auditory stimuli). Each sound clip was from one of the eight sound categories: acoustic guitar, choir, movie soundtracks (two), electric piano, electric guitar, synthesizer, and an orchestra. Each sound category consisted of 24 sound clips. Each sound within a sound category was randomly assigned to a phase offset modulation with the constraint that the number of sounds for each phase offset modulation was equal. Each movie clip was randomly paired with a sound. The random assignment of phase offset modulation to a sound and a movie to a sound was done before the experiment and consistent across all the participants.

The experimental apparatus and stimulus presentation were identical to that used by Clouter et al. (2017), their Experiment 3. The experiment was programmed with MATLAB (R2013a; MathWorks) using the Psychophysics Toolbox extensions (Brainard, 1997; Pelli, 1997; Kleiner et al., 2007). Presentation of visual stimuli were on a 21 inch CRT display with an nVidia Quadro K600 graphics card ( $875 \mathrm{MHz}$ graphics clock, 1024 MB dedicated graphics memory; NVidia). Participants sat $\sim 60 \mathrm{~cm}$ from the center of the monitor. The screen refresh rate of the monitor was $75 \mathrm{~Hz}$. Auditory stimuli were presented with insert earphones (ER3C, Etymotic Research).

Experimental procedures. Following the provision of informed consent, participants were prepared for EEG data collection, and were given instructions for, and familiarized with the tasks. During the formal experiment, participants were monitored by a web camera connected to a monitor in an adjoining EEG control room.

The experiment consisted of 12 blocks of an associative memory task, followed by synchrony judgment and unimodal source localizer tasks (see the last two paragraphs of this section). Each associative memory task block comprised an encoding phase, a distractor phase and an associative memory recall test phase (Fig. 2). On each trial during the encoding phase, participants were presented with 1 of 16 movies along with 1 of 16 sound clips. Each trial started with a fixation cross with an intertrial interval between 1 and $3 \mathrm{~s}$. Then, a sound-movie pair was presented for $3 \mathrm{~s}$. After the presentation, participants were instructed to make a judgment as to how well the sound suited the contents of the movie by pressing the number keys on a keyboard. The instruction screen was presented until a response was made. The ratings ranged from 1 (the sound does not suit the movie at all) to 5 (the sound suits the movie very well). Participants were instructed to remember the association between the sound and the movie within each trial, upon which their memory would later be tested. Within each block, four sounds from four categories were associated randomly with the 16 movies with the constraint that the number of sounds for each phase offset condition was equal (i.e., two $0^{\circ}$ phase offset modulated sounds and two $180^{\circ}$ phase offset modulated sounds in one sound category).

The distractor phase was presented after the last trial of the encoding phase. Participants were presented with a random number on the screen, drawn randomly from 170 to 199 . Participants were instructed to count backward, aloud, by 3 starting from this number, for $30 \mathrm{~s}$. 
A

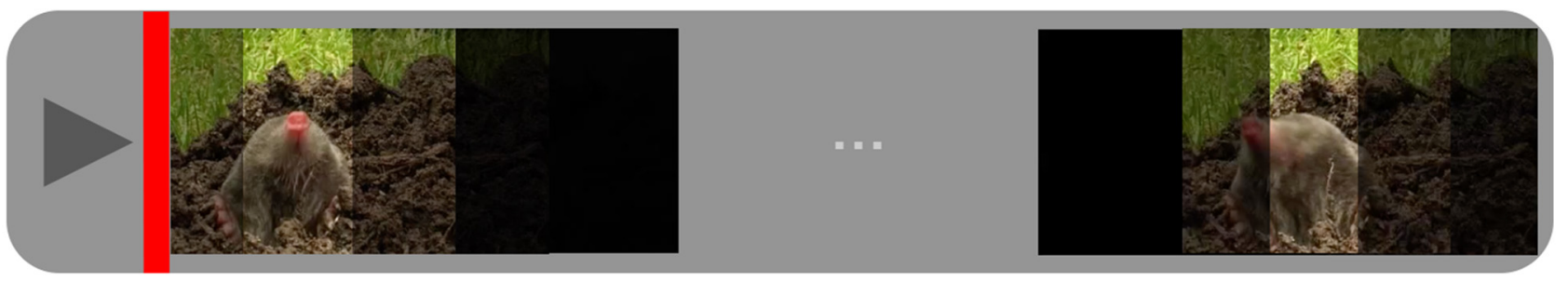

$\begin{array}{ll}0 & 0.125\end{array}$

2.75

$3 s$

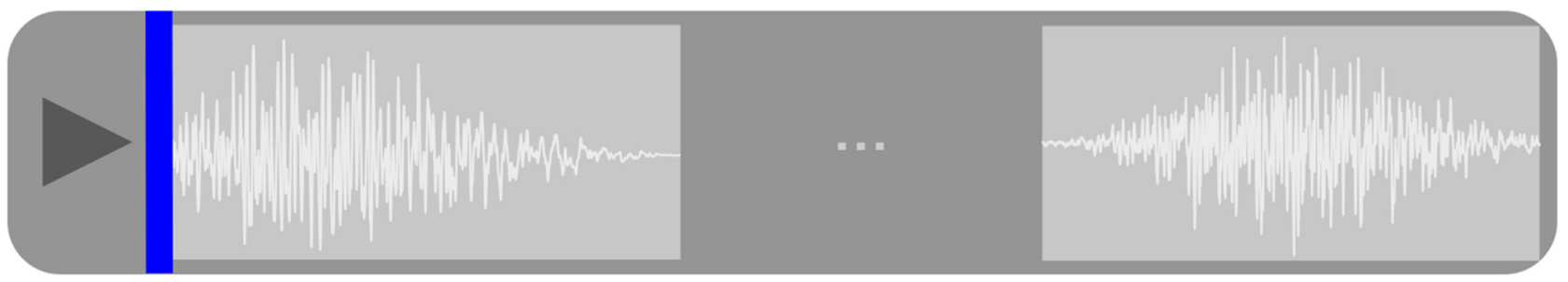

B

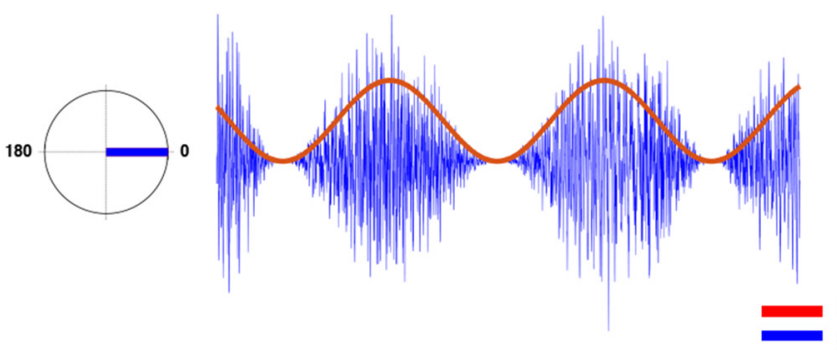

phase offset 0

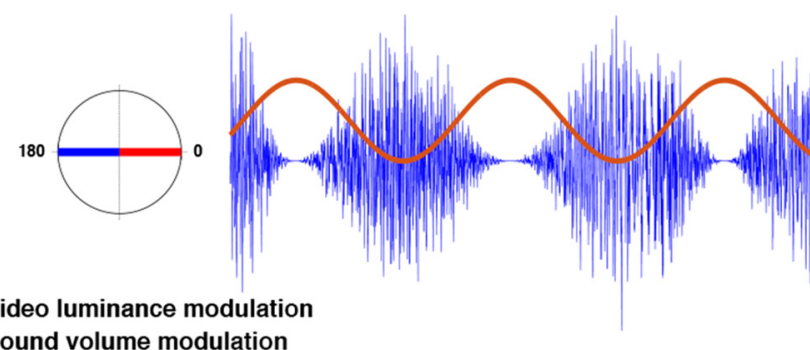

phase offset 180

Figure 1. Stimulus material of associative memory encoding task. $A$, An example of visual and auditory stimuli presented in the associative memory encoding task. Top, Movies were luminance modulated with a sine wave, and so flickered visually at $4 \mathrm{~Hz}$. Bottom, Sounds were amplitude modulated with a sine wave, and "flickered" at $4 \mathrm{~Hz}$. The movie and the sound are flickering in phase (i.e., with a $0^{\circ}$ phase offset). $B$, Sine-wave-modulated visual (red) and auditory (blue) stimuli. Left, The sound clip was amplitude modulated at $0^{\circ}$ phase offset from the sine wave that modulated the luminance of the movie. Right, The sound clip was amplitude modulated at $180^{\circ}$ phase offset from the sine wave that modulated the luminance of the movie. Note, that the auditory stimulus always lagged $40 \mathrm{~ms}$ with respect to the visual stimulus, which is not shown here for illustration purposes.

Participants were prompted on the screen to stop the distractor phase and start the associative memory recall test phase. In each block, the associative memory recall test phase consisted of 16 test trials, thus testing all of the 16 associations learned during the encoding phase. Each trial started with a fixation cross with an intertrial-interval between 1 and $3 \mathrm{~s}$. Participants were presented with 1 of the 16 sounds heard during the encoding phase for $3 \mathrm{~s}$, along with four still images from four of the movies from the encoding phase. Then, participants were instructed to select the movie that was presented with the sound in the encoding phase using the number keys 1 through 4 . The instruction screen was presented until a response was made. The movies from which to choose were, in the encoding phase, all presented with a sound from the same sound category.

The block presentation order was counterbalanced to ensure that each block was presented in each serial position an equal number of times across participants. Participants were allowed to take a break, if required, following each block. After the completion of the 12 associative memory task blocks, participants were given additional instructions for a synchrony judgment task. In this task participants were presented with 24 sound-movie pairs, drawn from the 192 sound-movie pairs. During the synchrony judgment task subjects were asked whether they could detect the synchrony (in-phase, $0^{\circ}$ phase offset) or asynchrony (out-of-phase, $180^{\circ}$ phase offset) between the flicker of the movie and sound. Participants were instructed to indicate asynchrony pressing number key 1 and indicate synchrony pressing 2 .
Last, two unimodal source localizer tasks were run. Each consisted of 50 trials of flickered sound clips only, or 50 trials of flickered movie clips only. The sound and movie clips were drawn from the 192 sounds and movies used throughout the experiment. On each trial, participants were asked to rate how pleasant the sound or movie was by using the number keys 1 (the sound or the movie was very unpleasant) through 5 (the sound or the movie was very pleasant).

EEG recordings and preprocessing. EEG was recorded from 128 scalp channels using a BioSemi ActiveTwo system. Vertical eye movements were recorded from an additional electrode placed $1 \mathrm{~cm}$ below the left eye. Horizontal eye movements were recorded from two additional electrodes placed $1 \mathrm{~cm}$ to the left of the left eye and to the right of the right eye. Online EEG signals were sampled at $1024 \mathrm{~Hz}$ by using the BioSemi ActiView software. The positions of each participant's electrodes were tracked using a Polhemus FASTRAK device (Colchester) and recorded by Brainstorm (Tadel et al., 2011) implemented in MATLAB.

Offline EEG data were preprocessed using the Fieldtrip toolbox for MEG and EEG analysis (Oostenveld et al., 2011). The continuous EEG data were bandpass filtered between 1 and $100 \mathrm{~Hz}$ and bandstop filtered between $48-52$ and $98-102 \mathrm{~Hz}$ to remove potential line noise at 50 and $100 \mathrm{~Hz}$. The data were then epoched from $2000 \mathrm{~ms}$ before stimulus onset to $5000 \mathrm{~ms}$ after stimulus onset, and downsampled to $512 \mathrm{~Hz}$. Bad channels and trials with coarse artifacts were excluded by visual inspection before applying an independent component analysis (ICA). In the unimodal conditions, one bad channel was excluded for each of five partic- 


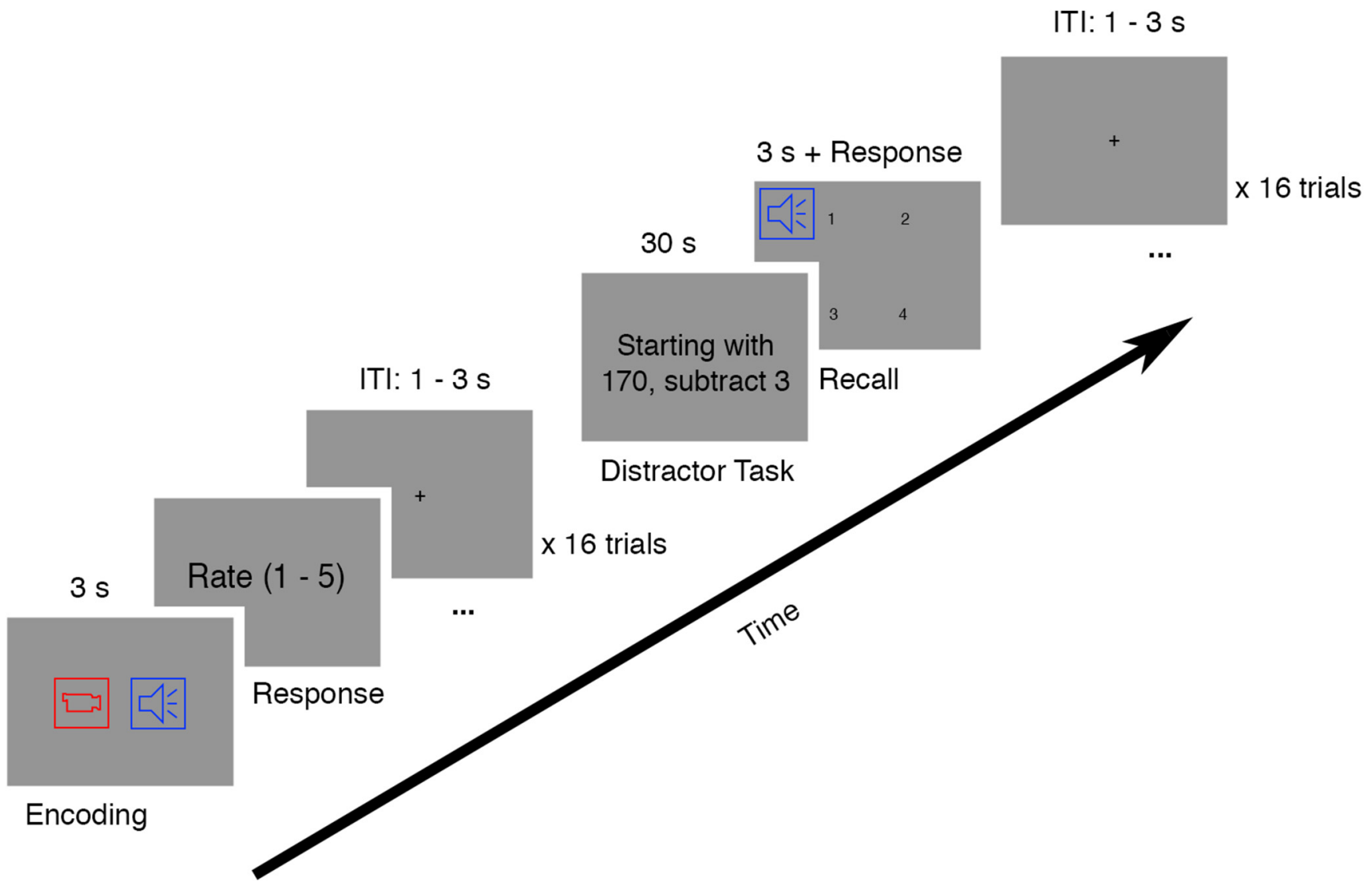

Figure 2. Experimental procedure. For each encoding trial, a 3 s sound-movie pair was presented. Participants were instructed to make a judgment as to how well the sound suited the contents of the movie after the presentation of the pair. After 16 encoding trials, a distractor task was presented. Participants were instructed to count backward in steps of three starting from a random number for $30 \mathrm{~s}$. Participants were prompted to stop the distractor task and start a recall trial. Participants were presented with one of the 16 sounds heard during the encoding phase for $3 \mathrm{~s}$ while four still images from four of the movies from the encoding phase were presented on the screen. Participants were instructed to select the movie that was presented with the sound in the encoding phase. The recall test phase also consisted of 16 trials hence testing all of the 16 associations studied in the encoding phase.

ipants. Four bad channels were excluded for another one participant. In the multimodal conditions, one bad channel was excluded for each of three participants. Two bad channels were excluded for another one participant. Four bad channels were excluded for each of another two participants. ICA components suggesting eye movement artifacts and regular pulse artifacts were removed from the data. Bad channels were interpolated by the method of triangulation of nearest neighbors based on the individuals' electrode positions. After re-referencing the data to average, trials with artifacts were manually rejected by visual inspection. Participants with $<16$ artifact-free trials in any of the conditions of interest were excluded from further analysis. The mean number of trials remaining in each condition were as follows: unimodal sound: 42 (25$50)$, unimodal movie: $42(26-49)$, multimodal 0 remembered: 34 (2148), multimodal 0 forgotten: 43 (32-55), multimodal 180 remembered: 31 (22-44), multimodal 180 forgotten: $46(28-61)$.

For three participants who provided their own MRI scans, their head models were created based on their structural scans (cf. Michelmann et al., 2016) The MRI scans were segmented into four layers, brain, CSF, skull, and scalp by using the Statistical Parametric Mapping 8 (SPM8) toolbox (http://www.fil.ion.ucl.ac.uk/spm) and the Huang toolbox (Huang et al., 2013). Then a volume conduction model was constructed by calling the "dipoli" method implemented in the Fieldtrip toolbox. Individuals" electrode positions were aligned to their head models. For further group analysis across all 24 participants, each individual MRI was warped to an MNI template MRI provided by the Fieldtrip toolbox then the inverse of the warp was applied to a template dipole grid to have a same location of each grid point for each participant in normalized MNI space. For the remaining participants who did not have their own MRI scans, the MNI template MRI and a template volume conduction model were used. In- dividuals' electrode positions were aligned to the template head model. Source models were prepared with the template volume conduction model and the aligned individuals' electrode positions.

Unimodal source localization. In the unimodal sound condition, EEG data from the unimodal sound condition was scalp current density (SCD) transformed using the finite-difference method (Huiskamp, 1991; Oostendorp and van Oosterom, 1996). In addition, the leadfields that were computed based on scalp potentials were SCD transformed by applying the transformation matrix that was used for transforming the potential data. This step is necessary for source localization and reconstruction of data from correlated with brain sources (such as data from auditory sources) using the beamforming method (Murzin et al., 2013).

Source activity was reconstructed using a linearly constrained minimum variance (LCMV) beamforming method (Van Veen et al., 1997). In the movie condition, source analysis was calculated for individual electrode positions, individual volume conduction model and normalized grid positions for the three participants who had their MRI scans. For the other participants, source analysis was run with individual electrode positions, grid positions and template volume conduction model. All the analyses were run on potential (i.e., average referenced) data. In the sound condition, source analysis was conducted using SCD-transformed leadfields on SCD-transformed data. Time series data were reconstructed on 2020 virtual electrodes for each participant. Event-related potential (ERP) was calculated at each virtual electrode for each unimodal condition. Time-frequency analysis was applied to each source ERP with a Morlet wavelet (width $=7$ ) using a frequency of interest between 3 and 5 $\mathrm{Hz}$. Evoked power was averaged between 0.75 and $2.75 \mathrm{~s}$ poststimulus onset and between 3.5 and $4.5 \mathrm{~Hz}$. A baseline condition was created by randomly assigning each trial to $0,90,180$, or $270^{\circ}$ phase offset by moving 
the signal onset forward in time by $0,32,64$, or 96 samples $(0,62.5,125$, or $187.5 \mathrm{~ms}$ ) with a restriction that the number of trials in each phase offset were approximately equal. The evoked power of the baseline condition was therefore expected to be minimal at corresponding auditory and visual sources. The evoked power differences were grand averaged across participants and the grand average evoked power differences were interpolated to the MNI MRI template. The coordinates for auditory and visual regions-of-interest (ROIs) were determined by where the maximum grand average evoked power differences were shown.

Multimodal source reconstruction. To reconstruct the time series data from auditory sources, the multimodal data were SCD-transformed. Beamforming analysis has a notoriously poor performance in reconstructing highly correlated source activity such as during auditory stimulation. A solution for EEG source localization was proposed by Murzin et al. (2013) who suggest that source analysis should be computed based on the scalp electrodes over left and right hemispheres separately. As a result, the spatial filter that was computed with only left scalp electrodes was applied to the left scalp time series data and the spatial filter that was computed with only right scalp electrodes was applied to the right scalp time series data. The time series data therefore corresponded to the sources of the left hemisphere and right hemisphere, respectively. The time series data at the left auditory ROI was extracted at the predetermined left auditory coordinate from the unimodal source localization step. Similarly, data at the right auditory ROI was extracted at the predetermined right auditory coordinate. The signals were then averaged across left and right auditory ROIs. The time series data at visual ROI was reconstructed without SCD transformation and was extracted at the predetermined visual coordinate.

To solve the problem of random direction of source dipoles caused by the LCMV beamformer, each participant's left and right auditory source ERPs were plotted along with visual source ERPs in each phase offset condition. The sign of the source reconstructed time series data were flipped in direction if any source ERPs showed the opposite of the expected direction of the visual and auditory ERP components $(\mathrm{P} 1, \mathrm{~N} 1$, or P2) by multiplying the data in the time series by -1 . The same approach was taken if the time series showed the opposite of the expected entrainment (with respect to the flicker of the physical stimuli; e.g., if the $0^{\circ}$ phase offset condition looked like $180^{\circ}$, or the $180^{\circ}$ phase offset condition looked like $0^{\circ}$ ). This flipping procedure was applied consistently across all trials, regardless of condition. A control analysis was conducted to confirm that the phase relationships between $0^{\circ}$ and $180^{\circ}$ conditions in the auditory and visual ROIs remained constant between before and after flipping. All distributions were non-uniform as indicated by the Rayleigh test. Thus the flipping procedure only resulted in a better illustration of the entrainment effect but did not bias the results in favor of our hypothesis.

Experimental design and statistical analysis. The experiment was within-subject design. To test the replication of the behavioral effect of phase offset condition $\left(0^{\circ}\right.$ vs $\left.180^{\circ}\right)$ on associative memory performance, a paired-samples $t$ test was used. To compute the instantaneous phase differences, the source reconstructed time series data were bandpass filtered between 1.5 and $9 \mathrm{~Hz}$. Then, the ERPs were computed for each phase offset condition at each source. The Hilbert transformation was applied to the source grand averaged ERPs that were redefined in time (1000 ms before stimulus onset to $4000 \mathrm{~ms}$ after stimulus onset). The instantaneous phases were calculated from the Hilbert transformed data and unwrapped. The instantaneous phase differences were calculated between auditory and visual sources for $1 \mathrm{~s}$, beginning $1 \mathrm{~s}$ after stimulus onset (to avoid influences of onset and offset responses) in each phase offset condition. The Rayleigh test and V test were used to test circular uniformity of the instantaneous phase differences in each phase offset condition.

For the subsequent memory analysis, the Hilbert transformation was applied to the filtered single-trial source data. The instantaneous phases of each trial were calculated from the Hilbert transformed data and unwrapped. The instantaneous phase differences were calculated between auditory and visual sources for $1 \mathrm{~s}$, beginning one second after stimulus onset. The single-trial phase entrainment measure in each phase offset condition was computed for each trial and each time point by calculating the resultant vector length of a vector that consisted of the data value at the time point and the theoretical phase offset $\left(0^{\circ}\right.$ or $\left.180^{\circ}\right)$. The resultant vector length was collapsed across time between 1 and $2 \mathrm{~s}$, resulting in one value per trial. A 2 (phase offset condition) $\times 2$ (subsequent memory) repeated-measures ANOVA was conducted on the averaged single-trial phase entrainment values for remembered and forgotten items in each phase offset condition.

To further test whether the degree of single-trial phase entrainment predicts subsequent memory performance, a logistic regression model was fit using the single-trial phase entrainment measure as the predictor, and memory performance on each trial as the dependent variable, resulting in model slopes for each phase offset condition. These slopes were normalized for each participant. The normalized slopes were then averaged between the two phase offset conditions for each participant. A one sample $t$ test was conducted to test whether the slopes were significantly $>0$.

To get a more fine-tuned picture of the relationship between singletrial phase angle and memory performance, we calculated the mean phase direction of the time points between 1 and $2 \mathrm{~s}$ for each trial. Depending on the mean phase direction of a trial, we sorted the trials into four bins, centered at $0,90,180$, and $270^{\circ}$ each with a bin width of $\pm 45^{\circ}$. To reduce the trial-number bias in each bin, we randomly selected 12 trials (the minimum trial number was 13) from each bin and calculated the proportion of remembered trials of the 12 trials in each bin. This procedure was repeated 10 times and averages across these 10 repetitions were built for each subject to reflect the proportion of remembered trials in each phase bin. A repeated-measures ANOVA was conducted on the memory accuracy across the four bins.

\section{Results}

\section{Behavioral performance}

We replicated the previous findings (Clouter et al., 2017) by showing that memory performance in the $0^{\circ}$ phase offset condition was significantly better than in the $180^{\circ}$ phase offset condition (Fig. 3) using a paired-samples $t$ test; $t_{(23)}=2.069, p=0.025$ (one-sided). To rule out the influence of perceptual factors on the memory effect, a sensitivity index $\left(d^{\prime}\right)$ was computed for perceptual judgment performance on synchronous and asynchronous stimuli. One-sample $t$ tests showed that the $d^{\prime}$ for participants' judgment on synchronous stimuli versus asynchronous stimuli did not differ from zero (synchronous: $t_{(23)}=1.317, p=0.201$; asynchronous: $\left.t_{(23)}=1.008, p=0.324\right)$. In addition, a pairedsamples $t$ test showed that $d^{\prime}$ for discriminating synchronous from asynchronous stimuli did not differ from $d^{\prime}$ for discriminating asynchronous stimuli from synchronous stimuli; $t_{(23)}=$ $0.074, p=0.942$. These results suggest that participants were unable to discriminate synchronous stimuli from asynchronous stimuli, thus ruling out the contribution of basic perceptual confounds to the observed memory effect.

To investigate whether participants' subjective judgment on how well a sound suited the contents of a movie influenced memory performance, a 2 (subsequent memory) $\times 2$ (phase offset condition) repeated-measures ANOVA was performed on the average rating score. A significant main effect of subsequent memory was found (mean rating score in subsequently remembered items: 3.038, mean rating score in subsequently forgotten items: $\left.2.827, F_{(1,23)}=24.224, p<0.001\right)$. A significant main effect was also shown for phase offset, mean rating score in $0^{\circ}$ phase offset condition, 2.979 , mean rating score in $180^{\circ}$ phase offset condition, 2.886, $F_{(1,23)}=7.962, p=0.010$. These results suggest that the observed better memory performance for $0^{\circ}$ phase offset condition compared with $180^{\circ}$ condition could be driven by perceived semantic congruency between multisensory stimuli. To control for this possible alternative, we conducted an analysis where the trial numbers in each rating scale were equalized between the $0^{\circ}$ 
phase offset condition and the $180^{\circ}$ phase offset condition by randomly subselecting trials for each participant (mean trial number 170, range 148-190). Associative memory accuracy was calculated based on these selected trials, now equalized for semantic congruency, for each participant. This procedure was repeated 10 times. The resultant associative memory accuracy was averaged for each participant across 10 repetitions. The memory advantage in the $0^{\circ}$ phase offset condition was still valid, as supported by a paired-samples $t$ test; $t_{(23)}=1.903, p=$ 0.035 (one-sided), thus confirming our original results.

\section{Phase in sensory cortices is entrained to} multisensory stimulation

The ROIs obtained from the unimodal source localization results are shown in Figure 4. Multimodal grand average ERP waveforms were extracted from the visual and auditory ROIs determined by these unimodal source localization results. Figure 5 shows the grand average waveforms for the 0 and $180^{\circ}$ phase-offset conditions for the visual and auditory ROIs. Rayleigh tests and $\mathrm{V}$ tests were conducted for the $0^{\circ}$ and $180^{\circ}$ phase offset conditions separately, using the data points of instantaneous phase differences between auditory and visual signals from 1 to $2 \mathrm{~s}(n=513$ samples) as dependent variable. Both tests enable rejection of the null hypothesis that the distribution of the phase differences in the $0^{\circ}$ phase offset condition or the $180^{\circ}$ phase offset condition are uniformly distributed; the resultant vector length in the $0^{\circ}$ condition: $0.9197, p \approx 0$; resultant vector length in $180^{\circ}$ condition: $0.9192, p \approx$ 0 . The $\mathrm{V}$ test further suggests that the distribution of phase differences in each phase offset condition has a mean direction of its entrained phase, 0 or $180^{\circ}$ (both $p$ values $=0)$, respectively. These results suggest that our paradigm was effective in synchronizing $\left(0^{\circ}\right.$ condition $)$ or desynchronizing $\left(180^{\circ}\right.$ condition) the auditory and visual cortex in the theta frequency, replicating Clouter et al. (2017).

\section{Single-trial phase entrainment predicts subsequent memory performance}

To investigate our main hypothesis, i.e., whether phase differences between auditory and visual regions predict the likelihood of memory formation, a single-trial phase entrainment measure was calculated for each phase offset condition (see Materials and Methods). To this end the resultant vector length for every time point was calculated between the observed phase difference (between the auditory and visual cortices) and the phase difference of the physical stimuli (i.e., either 0 or $180^{\circ}$ ). This measure yields values close to 1 for high entrainment, where a given trial closely followed the rhythconditions is shown in gray.

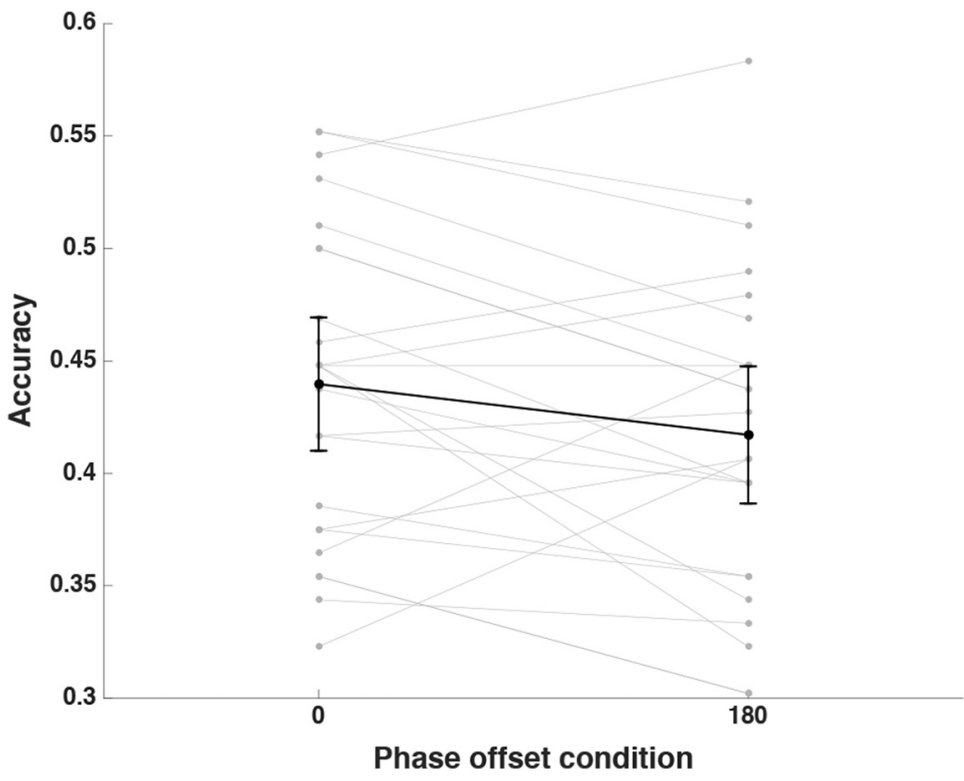

Figure 3. Associative memory task performance. Proportion of the correctly selected movie scenes that were associated with presented sounds in each phase offset condition. Note that chance level is at $25 \%$. Error bars represent $95 \%$ confidence intervals of means in 0 and 180 phase offset conditions. Individual data for correct associative memory performance in 0 and 180 phase offset

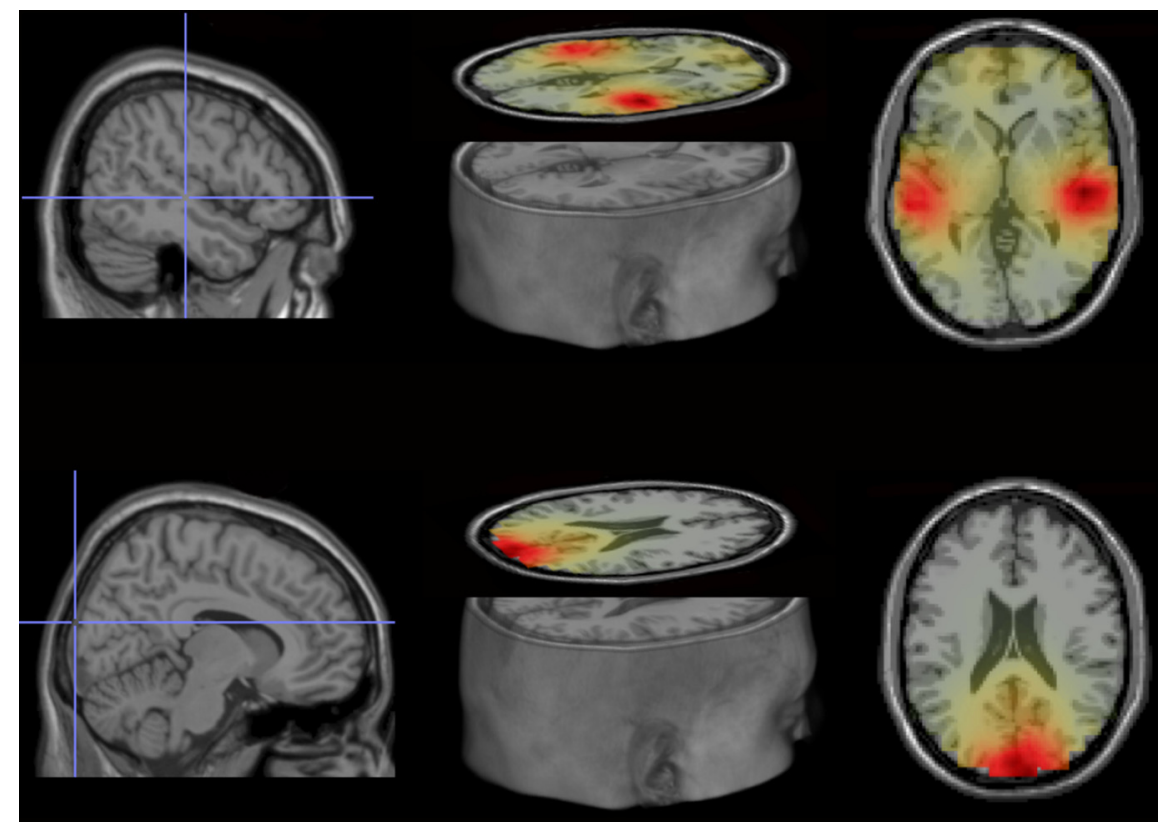

Figure 4. Source localization of theta power in the unimodal conditions. Top, Auditory sources, MNI coordinates of ROls: right, 50, -21 , 0; left, $-60,-29,0$. Bottom, Visual source, MNI coordinates of R0l: 10, $-99,20$. Evoked power was averaged over $3.5 \mathrm{and} 4.5 \mathrm{~Hz}$, between 0.75 and $2.75 \mathrm{~s}$ at each virtual electrode in the unimodal movie and sound conditions and the baseline conditions (see Materials and Methods). Grand average power differences between unimodal conditions and baseline conditions were interpolated to a MNI MRI template. The source coordinates were determined by where the maximum grand average power differences were. The source plots were generated by the Fieldtrip toolbox and MRIcro for OSX (http://www.mccauslandcenter.sc.edu/crnl/mricro).

mic stimulation, and values close to 0 for low entrainment, where a given trial deviated from the rhythmic stimulation. Figure 6 illustrates this measure with an example from four single trials. Figure $6, A$ and $B$, shows single trials from $0^{\circ}$ offset condition. The trial for a later-remembered stimulus reveals a strong entrainment at $0^{\circ}$, whereas the forgotten trial shows a small value. In contrast, the forgotten trial in the $180^{\circ}$ offset condition shows a strong entrainment to $180^{\circ}$, whereas the remembered trial shows 

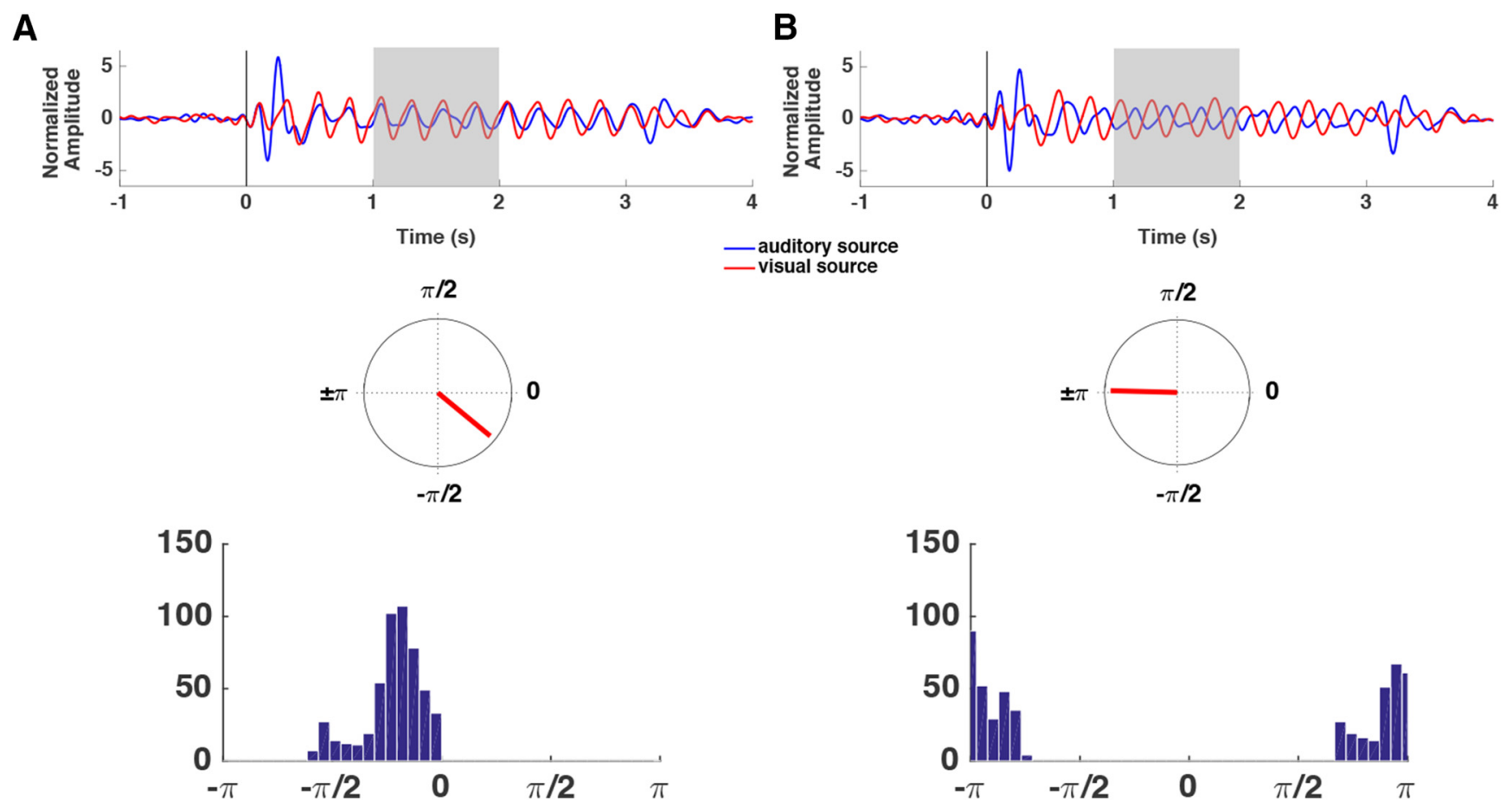

Figure 5. Phase differences between auditory and visual sources in each phase offset condition. $A$, Phase differences between auditory and visual sources in $0^{\circ}$ offset condition. Top, Amplitude normalized grand average ERP signals at auditory (blue) and visual sources (red). Middle, Mean resultant vector of instantaneous phase differences between auditory and visual sources, between 1 and $2 s$ (shaded time window on the grand average ERPs) is plotted on a unit circle. Bottom, Histogram of wrapped instantaneous phase differences between auditory and visual sources, between 1 and 2 s, using 40 equally-sized bins. $\boldsymbol{B}$, As in $\boldsymbol{A}$, but for the $180^{\circ}$ offset condition.

a weak entrainment to $180^{\circ}$ (Fig. 6C,D). This pattern is consistent with the notion that $0^{\circ}$ is optimal and $180^{\circ}$ is non-optimal for the formation of associative memories. To test this idea formally, we conducted a $2 \times 2$ ANOVA with the factors phase offset condition $\left(0\right.$ vs $\left.180^{\circ}\right)$ and subsequent memory (hits vs misses), expecting to find a significant interaction. Indeed, such a significant interaction between phase offset condition and subsequent memory was observed; $F_{(1,23)}=4.627, p=0.042$. Subsidiary paired-samples $t$ tests showed that this interaction was the result of a trend for stronger phase entrainment in remembered trials than forgotten trials in the $0^{\circ}$ phase offset condition; $t_{(23)}=1.496$, $p=0.074$ (one-sided), and significantly weaker entrainment in remembered trials than in forgotten trials in the $180^{\circ}$ phase offset condition; $t_{(23)}=-2.165, p=0.021$ (one-sided; Fig. $7 A$ ). Together, these results reveal that the likelihood of a movie and a sound being successfully associated in memory varies with the strength of entrainment. Hits were associated with strong entrainment in the $0^{\circ}$ phase offset condition but low entrainment in the $180^{\circ}$ phase offset condition, whereas misses showed the opposite pattern.

Another way to describe this interaction is that the closer the audio-visual phase difference in a given trial is to 0 , the higher the likelihood that the sound and the movie are associated in memory. To test this prediction more directly we measured the singletrial phase entrainment to $0^{\circ}$ in both phase offset conditions. We then fitted a logistic regression model by using single-trial phase entrainment to $0^{\circ}$ to predict memory outcome in each phase offset condition for each participant. The normalized slopes of each participant were averaged across two phase offset conditions. As shown in Figure 7B, one-sample $t$ tests revealed that the values of the slopes were significantly positive; $t_{(23)}=1.958, p=$ 0.031 (one-sided). This result indicates that associative memory performance increases as a function of synchronization between the visual and auditory cortex, i.e., the closer their phase difference is to $0^{\circ}$.

To illustrate this relationship between audio-visual synchronization and memory performance in a more fine-grained manner, single trials were sorted into four bins centered at $0,90,180$, and 270 . To account for possible trial-number bias, we equalized the number of trials in each bin by randomly selecting 12 trials from each bin. Then the proportion of remembered trials was calculated for each bin with this procedure repeated 10 times for each participant. Finally, the proportion of remembered trials calculated at each iteration was averaged across those 10 iterations. The results show that the recall accuracy in the $0^{\circ}$ bin was significantly higher than the accuracy in any of the other three bins (Fig. 7C), which did not differ from each other. A repeatedmeasures ANOVA with the factor phase bin $\left(0,90,180,270^{\circ}\right)$ statistically confirmed this effect on recall accuracy, $F_{(3,69)}=$ $6.014, p=0.001$. Pairwise $t$ tests showed that accuracy in the $0^{\circ}$ bin was significantly higher than in the 90,180 , and $270^{\circ}$ bins; $t_{(23)}=3.061, p=0.006 ; t_{(23)}=2.739, p=0.012 ; t_{(23)}=4.293, p<$ 0.001 . Whereas recall accuracy in the 90,180 , and $270^{\circ}$ bins did not statistically differ from each other (all $p s>0.8$ ). On average the difference between the $0^{\circ}$ phase bin and the other phase bins is $8 \%$. The effect size (as calculated with partial eta squared, $\eta_{\mathrm{p}}^{2}$ ) is 0.207 , based upon $F_{(3,69)}=6.014$, thus indicating a large effect size (Richardson, 2011). Although it is unlikely that a patient would subjectively notice such a change in memory performance (Frerichs and Tuokko, 2006) this effect might be of potential clinical interest for dementia research, as a study by Parra et al. (2009) showed that memory in Alzheimer's patients suffers the most for bound relative to single-feature objects (each of the two bound features). More generally, this result replicates the behavioral findings by Clouter et al. (2017), and suggests further that 
A

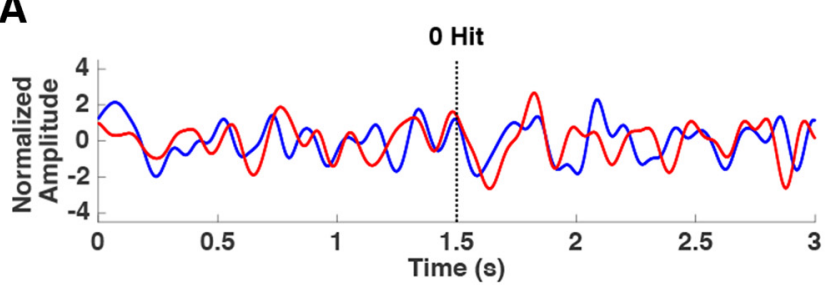

B

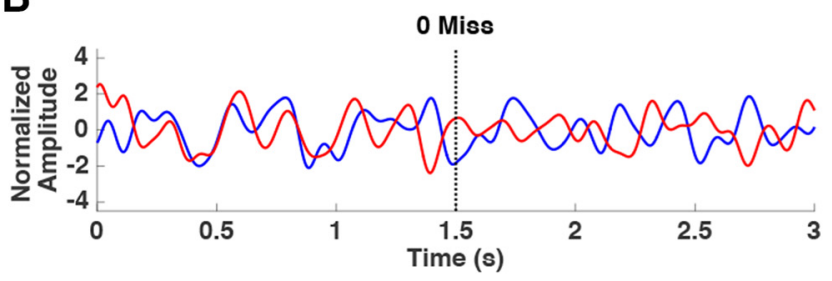

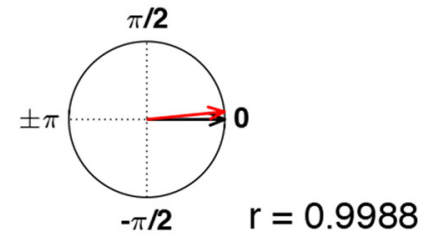

C
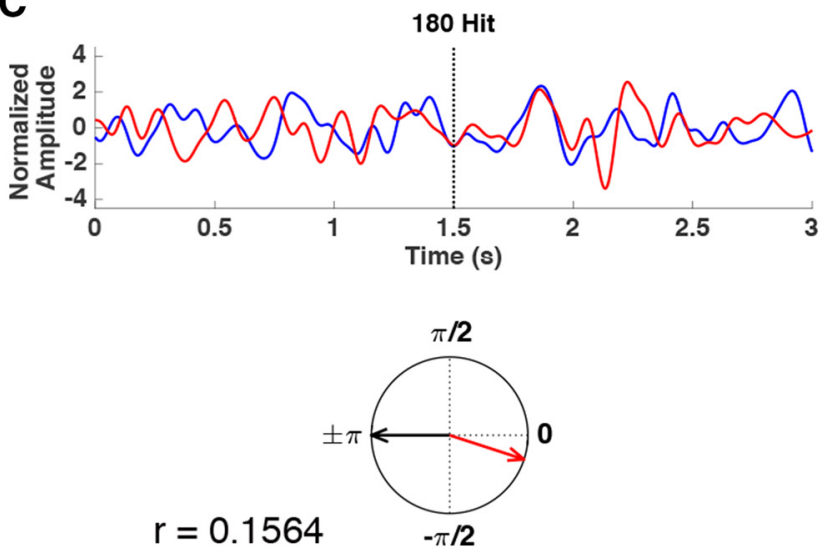

- auditory source - visual source

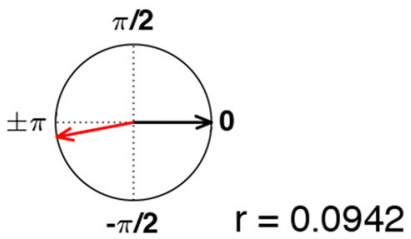

D
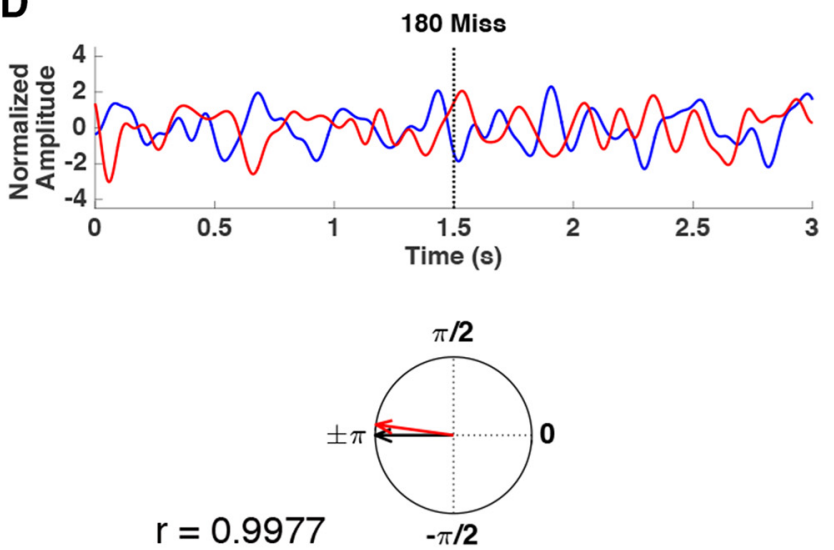

Figure 6. An example of four single trials in $0^{\circ}$ remembered, forgotten conditions and $180^{\circ}$ remembered, forgotten conditions, respectively. $\boldsymbol{A}$, Top, A bandpass filtered (1.5-9 $\mathrm{Hz}$ ) single trial from auditory (blue) and visual (red) sources in $0^{\circ}$ offset remembered condition. Amplitude was normalized. Bottom, Instantaneous phase difference between auditory and visual sources at a certain time point $1.5 \mathrm{~s}$ of the single trial is plotted on a unit circle (red line). Single-trial phase entrainment was calculated as the resultant vector length between the measured phase difference (red arrow) and the entrained phase offset $0^{\circ}$ (black arrow), which was 0.9988 in this case. $\boldsymbol{B}$, Same as in $\boldsymbol{A}$ but for $0^{\circ}$ offset forgotten. $\boldsymbol{C}$, Same as in $\boldsymbol{A}$ but for a $180^{\circ}$ offset remembered trial. $\boldsymbol{D}$, Same as in $\boldsymbol{A}$ but for a $180^{\circ}$ offset forgotten trial.

there is a narrow time window around $0^{\circ}$ for phases to synchronize to optimize the formation of associative memories.

\section{Discussion}

We investigated a fundamental question in the field of memory research, i.e., does the degree of theta phase synchronization between visual and auditory cortical regions predict successful encoding of associations between visual and auditory information. We used a recently developed multisensory entrainment paradigm (Clouter et al., 2017) and replicated the findings from Clouter et al. (2017) by showing that episodic memory performance was significantly enhanced when the multisensory information was presented synchronously at $4 \mathrm{~Hz}$. We used the physical sensory stimuli required to be associated to entrain the respective visual and auditory cortices at the intended phase offsets, successfully replicating Clouter et al. (2017). Importantly, however, our results go beyond the previous findings by Clouter et al. (2017) to reveal that the single-trial phase synchrony between visual and auditory cortex predicts subsequent success on encoding the association between the sensory information into long-term memory. This finding is crucial because it reveals the underlying mechanism of synchronous presentation between sensory stimuli at theta frequency enhancing episodic memory. Moreover, the findings provide additional evidence as to the causal role of theta phase in human episodic memory formation by showing that synchronized theta activity between sensory in- formation processing areas is a critical mechanism to bind multisensory information into episodic memory on a trial-by-trial basis.

Computational models suggest that the hippocampus binds diverse cortical representations into a sparse unified episodic memory representation by rapid synaptic modifications between cortical inputs and hippocampal neurons through LTP (McClelland et al., 1995). The induction of LTP depends on the coordinated timing of action potentials across populations of neurons as demonstrated by the animal literature in vitro and in vivo that stimulation at the peak of hippocampal theta phase induces LTP (Pavlides et al., 1988; Huerta and Lisman, 1995; Hölscher et al., 1997; Hyman et al., 2003). Given the causal role of theta phase in the induction of LTP, the relative timing between cortical inputs and hippocampal theta oscillations should also play a causal role in human episodic memory formation. However, most evidence is correlational and post hoc (Fell et al., 2003; Rutishauser et al., 2010; Backus et al., 2016). Clouter et al. (2017) provided the first causal evidence in humans by showing that associative memory performance was significantly better in the $0^{\circ}$ condition than the $180^{\circ}$ condition. The present study successfully replicates the findings from Clouter et al. (2017) although our effect is weaker and with more variability. One possible reason is that half of our stimuli was not used by Clouter et al. (2017), which might cause variability in entraining visual and auditory regions, hence 

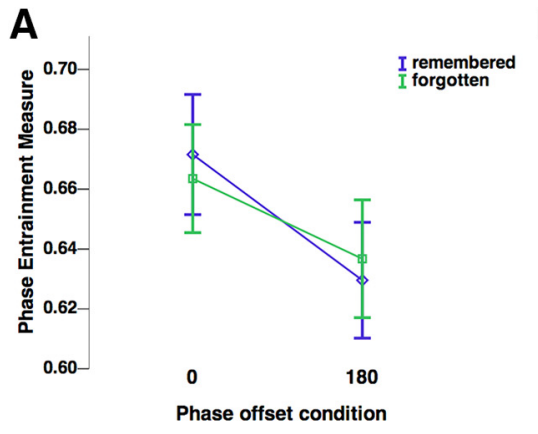

B

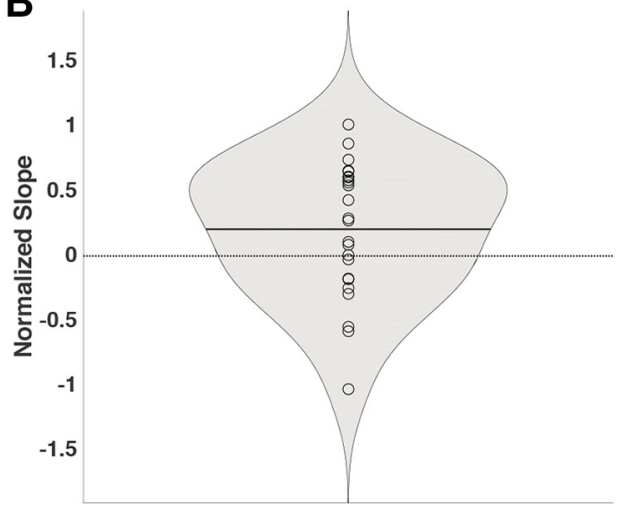

\section{C}

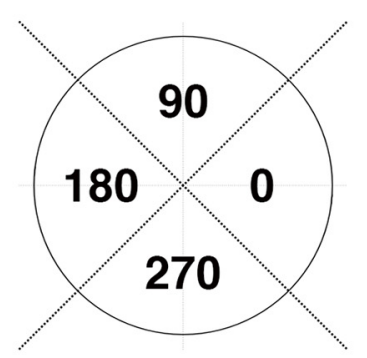

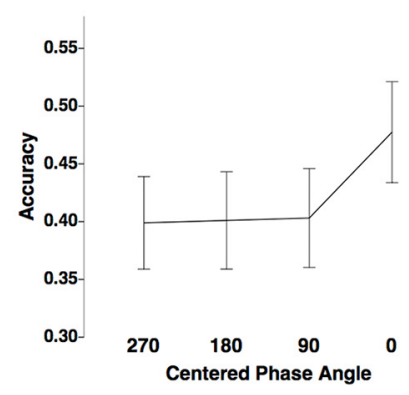

Figure 7. Memory performance as a function of phase entrainment. $\boldsymbol{A}$, Phase entrainment measure was averaged between 1 and $2 \mathrm{~s}$ for each trial. These single-trial phase entrainment values are plotted as a function of subsequent memory (remembered, purple; forgotten, green) in each phase offset condition. Error bars represent $95 \%$ confidence intervals of means in 0 remembered, 0 forgotten, 180 remembered, 180 forgotten conditions. $\boldsymbol{B}$, Normalized slopes of logistic regression models for each phase offset condition were averaged between conditions for each subject. The logistic regression model was fitted to each subject's memory performance, against the single-trial phase entrainment measure (averaged between 1 and $2 \mathrm{~s}$ ). The violin plot represents the distribution of the averaged normalized slope values. The black horizontal bars represent the mean of the data. Individual scatter points represent the averaged normalized slope values for each participant. Violin plots were created with the MATLAB extension violin.m (Hoffmann, 2015). C, Recall accuracy of each phase offset bin that consisted of trials which mean phase direction of each trial was $270,180,90$, and $0^{\circ}, \pm 45^{\circ}$ respectively. Left, An illustration of how the four phase offset bins were defined. Four bins were centered at $0,90,180,270^{\circ}$, with a bin boundary of $\pm 45^{\circ}$. Right, Recall accuracy was calculated in each bin. The $x$-axis represents the centered phase angle of each bin. For each trial, the mean phase direction between 1 and $2 \mathrm{~s}$ was computed. Then, each trial was sorted into one of the four bins depending on the mean direction (e.g., if the mean phase direction of a trial was a value between -45 and $45^{\circ}$, the trial would be sorted into the $0^{\circ}$ bin). The proportion of remembered trials was calculated for 12 trials that were randomly selected from each bin for each iteration. The recall accuracy was averaged across 10 iterations. Error bars represent $95 \%$ confidence intervals of means in each bin condition.

reducing the effect. However, this variability allowed us to link trial-by-trial fluctuation in phase entrainment with subsequent memory performance. The strength of single-trial entrainment in the $0^{\circ}$ condition was positively related to episodic binding between visual and auditory information in that trial. In contrast, for the $180^{\circ}$ condition stronger entrainment was related to a failure in memory formation. To put it another way, the closer the 4 $\mathrm{Hz}$ phase difference between the two sensory regions was to $0^{\circ}$ the better the memory outcome (Fig. $7 B$ ). This finding extends our previous results in that it directly links the theta phase dynamics with memory performance on a single-trial level, suggesting that the relative timing in theta neural oscillations is a main driving force underlying long-term associative memory formation.

Learning of associations between unrelated elements is highly dependent on the hippocampus (Gonzalo et al., 2000; Eichenbaum and Cohen, 2004; Staresina and Davachi, 2009). Although we were not able to observe signals from the hippocampus, the hippocampus is strongly implicated as the candidate region influencing the subsequent memory effect. Successful encoding of associations between cortical inputs happens when the inputs arrive at the peak of hippocampal theta phase triggering strong
LTP in the synapses (Hasselmo, 2005). In human studies, functional connectivity between neocortical areas and the medial temporal lobe (MTL) has been related to successful episodic encoding (Summerfield et al., 2006; Schott et al., 2013). Moreover, item-context binding and integration between new information and old mnemonic trace has been suggested to be supported functionally by MTL theta power, as well as theta coupling between the MTL and cortical regions (Staudigl and Hanslmayr, 2013; Backus et al., 2016). Therefore, the current finding that synchronization between visual and auditory theta activity predicts later memory success could reflect that coincident volleys of action potentials terminating in the MTL at similar theta phases, which is then more likely to induce hippocampal LTP in the synapses of the respective auditory and visual downstream neural ensembles in the MTL.

One could argue that the observed phase synchronization between early sensory areas reflects multisensory integration, which captures attention and results in better memory (Senkowski et al., 2008). However, the observed interaction between strength of entrainment and subsequent memory rules out the explanation that successful encoding of a soundmovie pair depends on early sensory level attention. The strength of entrainment to a periodic stimulus is positively related to attention, with attention increasing the entrainment response (Müller et al., 2006; Lakatos et al., 2008; Saupe et al., 2009; Nozaradan et al., 2012). If attention at the sensory level is the sole factor modulating successful memory formation, phase entrainment should be significantly stronger for remembered trials than forgotten trials, regardless of the phase offset condition (i.e., 0 vs 180), which is not what we observed. Furthermore, the interaction effect between subsequent memory and phase offset condition was shown only between 1 and $2 \mathrm{~s}$ after stimulus onset, not before or after. This time course suggests that the memory formation was not influenced by early evoked responses or stimulus-related phase coupling typically reported in the multisensory integration literature (Senkowski et al., 2008) but influenced by synchronized theta activity in a later and more sustained fashion, which is arguably a mechanism of successful encoding of associations (Summerfield and Mangels, 2005).

A remarkable finding is the fact that the degree of phase entrainment showed a reverse function of subsequent memory in the $180^{\circ}$ condition. This raises the question of what the underlying mechanism of the weaker entrainment to $180^{\circ}$ for remembered trials is. This perturbation from $180^{\circ}$ points toward a resilience mechanism in the brain, preventing it from being entrained to a non-optimal phase offset to get closer to a more optimal phase for forming a memory association (i.e., $0^{\circ}$ ). Hippocampo-cortical loops are plausible candidates for such a 
mechanism, which fits with fMRI findings showing that increases in hippocampal activity are correlated with spatiotemporal desynchronization between elements that form a memory (Staresina and Davachi, 2009). Therefore, the hippocampus might put in more work when encoding associations between stimuli that are asynchronously presented. However, how the hippocampus feeds back the cortical information so that it can avoid being entrained remains unknown. A possible explanation could be that the overall hippocampal theta power increases when phase difference gets closer to $0^{\circ}$ relative to when the inputs are strongly entrained to $180^{\circ}$, creates a "theta state" conducive to episodic binding. If this is the case, memory should also slightly benefit from $90^{\circ}$ and $270^{\circ}$ phase offsets. However, when sorting single trials into four phase bins corresponding to $0,90,180$, and $270^{\circ}$ memory performance in the $0^{\circ}$ condition was significantly better than in any of the other three conditions, with 90,180 , and $270^{\circ}$ showing equally poor performance levels. This pattern perfectly replicates the pattern obtained by Clouter et al. (2017) and suggests that there is a narrow time window around $<62.5 \mathrm{~ms}$ during which neural events have to coincide to result in effective memory formation. One such fine grained learning mechanism is spike-timing-dependent-plasticity (STDP; Song et al., 2000). However, whether STDP is involved in the observed effects needs to be further investigated.

In conclusion, our findings support the notion that externally induced interregional theta phase synchronization supports associative memory formation on a trial-by-trial basis. By successfully replicating findings from a previous study that used the same paradigm (Clouter et al., 2017), we further show that the degree of multisensory entrainment causes subsequent remembering and forgetting. Stronger entrainment in the $0^{\circ}$ condition leads to successful memory while stronger entrainment in the $180^{\circ}$ condition relates to failure in episodic binding. Such a finding supports that human episodic memory formation indeed depends on relative timing between the cortical processing of sensory streams, in turn providing a powerful tool to predict single-trial memory formation based on the performance of entrainment on any given trial. With this experimental paradigm, neural synchronization can be precisely controlled to test how synchronized neural activity influences recollection versus familiarity and monitor whether the current brain state is at encoding or retrieval (Rizzuto et al., 2006; Ezzyat et al., 2017), given the role of different hippocampal theta phases in episodic memory processes (Hasselmo et al., 2002; Hasselmo, 2005). Clinically, the paradigm could provide a noninvasive and accessible way to enhance episodic memory performance via external synchronization in healthy subjects and patients suffering from memory disorders such as Alzheimer (Huerta and Lisman, 1995; Iaccarino et al., 2016).

\section{References}

Backus AR, Schoffelen JM, Szebényi S, Hanslmayr S, Doeller CF (2016) Hippocampal-prefrontal theta oscillations support memory integration. Curr Biol 26:450-457. CrossRef Medline

Bliss TV, Collingridge GL (1993) A synaptic model of memory: long-term potentiation in the hippocampus. Nature 361:31-39. CrossRef Medline

Brainard DH (1997) The psychophysics toolbox. Spat Vis 10:433-436. CrossRef Medline

Buzsáki G (2010) Neural syntax: cell assemblies, synapsembles, and readers. Neuron 68:362-385. CrossRef Medline

Clouter A, Shapiro KL, Hanslmayr S (2017) Theta phase synchronization is the glue that binds human associative memory. Curr Biol 27:31433148.e6. CrossRef Medline

Eichenbaum H, Cohen NJ (2004) From conditioning to conscious recollection: memory systems of the brain. Oxford, UK: Oxford University Press.
Ezzyat Y, Kragel JE, Burke JF, Levy DF, Lyalenko A, Wanda P, O'Sullivan L, Hurley KB, Busygin S, Pedisich I, Sperling MR, Worrell GA, Kucewicz MT, Davis KA, Lucas TH, Inman CS, Lega BC, Jobst BC, Sheth SA, Zaghloul K, et al. (2017) Direct brain stimulation modulates encoding states and memory performance in humans. Curr Biol 27:1251-1258. CrossRef Medline

Fell J, Klaver P, Elfadil H, Schaller C, Elger CE, Fernández G (2003) Rhinalhippocampal theta coherence during declarative memory formation: interaction with gamma synchronization? Eur J Neurosci 17:1082-1088. CrossRef Medline

Frerichs RJ, Tuokko HA (2006) Reliable change scores and their relation to perceived change in memory: implications for the diagnosis of mild cognitive impairment. Arch Clin Neuropsychol 21:109-115. CrossRef Medline

Gonzalo D, Shallice T, Dolan R (2000) Time-dependent changes in learning audiovisual associations: a single-trial fMRI study. Neuroimage 11:243255. CrossRef Medline

Hasselmo ME (2005) What is the function of hippocampal theta rhythm? Linking behavioral data to phasic properties of field potential and unit recording data. Hippocampus 15:936-949. CrossRef Medline

Hasselmo ME, Bodelón C, Wyble BP (2002) A proposed function for hippocampal theta rhythm: separate phases of encoding and retrieval enhance reversal of prior learning. Neural Comput 14:793-817. CrossRef Medline

Hoffmann H (2015) violin.m: simple violin plot using MATLAB default kernel density estimation. Katzenburgweg, Germany: INRES (University of Bonn).

Hölscher C, Anwyl R, Rowan MJ (1997) Stimulation on the positive phase of hippocampal theta rhythm induces long-term potentiation that can be depotentiated by stimulation on the negative phase in area CA1 in vivo. J Neurosci 17:6470-6477. CrossRef Medline

Huang Y, Dmochowski JP, Su Y, Datta A, Rorden C, Parra LC (2013) Automated MRI segmentation for individualized modeling of current flow in the human head. J Neural Eng 10:066004. CrossRef Medline

Huerta PT, Lisman JE (1995) Bidirectional synaptic plasticity induced by a single burst during cholinergic theta oscillation in CA1 in vitro. Neuron 15:1053-1063. CrossRef Medline

Huiskamp G (1991) Difference formulas for the surface Laplacian on a triangulated surface. J Comput Phys 95:477-496. CrossRef

Hyman JM, Wyble BP, Goyal V, Rossi CA, Hasselmo ME (2003) Stimulation in hippocampal region CA1 in behaving rats yields long-term potentiation when delivered to the peak of theta and long-term depression when delivered to the trough. J Neurosci 23:11725-11731. CrossRef Medline

Iaccarino HF, Singer AC, Martorell AJ, Rudenko A, Gao F, Gillingham TZ, Mathys H, Seo J, Kritskiy O, Abdurrob F, Adaikkan C, Canter RG, Rueda R, Brown EN, Boyden ES, Tsai LH (2016) Gamma frequency entrainment attenuates amyloid load and modifies microglia. Nature 540:230235. CrossRef Medline

Jacobs J (2014) Hippocampal theta oscillations are slower in humans than in rodents: implications for models of spatial navigation and memory. Philos Trans R Soc Lond B Biol Sci 369:20130304. CrossRef Medline

Kleiner M, Brainard D, Pelli D, Ingling A, Murray R, Broussard C, others (2007) What's new in psychtoolbox-3. Perception 36:1. CrossRef Medline

Lakatos P, Karmos G, Mehta AD, Ulbert I, Schroeder CE (2008) Entrainment of neuronal oscillations as a mechanism of attentional selection. Science 320:110-113. CrossRef Medline

McClelland JL, McNaughton BL, O'Reilly RC (1995) Why there are complementary learning systems in the hippocampus and neocortex: insights from the successes and failures of connectionist models of learning and memory. Psychol Rev 102:419-457. CrossRef Medline

Michelmann S, Bowman H, Hanslmayr S (2016) The temporal signature of memories: identification of a general mechanism for dynamic memory replay in humans. PLOS Biol 14:e1002528. CrossRef Medline

Moscovitch M (2008) The hippocampus as a "stupid," domain-specific module: implications for theories of recent and remote memory, and of imagination. Can J Exp Psychol 62:62-79. CrossRef Medline

Müller MM, Andersen S, Trujillo NJ, Valdés-Sosa P, Malinowski P, Hillyard SA (2006) Feature-selective attention enhances color signals in early visual areas of the human brain. Proc Natl Acad Sci U S A 103:1425014254. CrossRef Medline 
Murzin V, Fuchs A, Scott Kelso JA (2013) Detection of correlated sources in EEG using combination of beamforming and surface Laplacian methods. J Neurosci Methods 218:96-102. CrossRef Medline

Muzzio IA, Kentros C, Kandel E (2009) What is remembered? Role of attention on the encoding and retrieval of hippocampal representations: attentional modulation of hippocampal representations. J Physiol 587:28372854. CrossRef Medline

Norman KA, Newman E, Detre G, Polyn S (2006) How inhibitory oscillations can train neural networks and punish competitors. Neural Comput 18:1577-1610. CrossRef Medline

Nozaradan S, Peretz I, Mouraux A (2012) Steady-state evoked potentials as an index of multisensory temporal binding. Neuroimage 60:21-28. CrossRef Medline

Oostendorp TF, van Oosterom A (1996) The surface laplacian of the potential: theory and application. IEEE Trans Biomed Eng 43:394-405. CrossRef Medline

Oostenveld R, Fries P, Maris E, Schoffelen JM (2011) FieldTrip: open source software for advanced analysis of MEG, EEG, and invasive electrophysiological data. Comput Intell Neurosci 2011:156869. CrossRef Medline

Parish G, Hanslmayr S, Bowman H (2018) The sync/deSync model: how a synchronized hippocampus and a de-synchronized neocortex code memories. J Neurosci 38:3428-3440. CrossRef Medline

Parra MA, Abrahams S, Fabi K, Logie R, Luzzi S, Della Sala S (2009) Shortterm memory binding deficits in Alzheimer's disease. Brain 132:10571066. CrossRef Medline

Pavlides C, Greenstein YJ, Grudman M, Winson J (1988) Long-term potentiation in the dentate gyrus is induced preferentially on the positive phase of theta-rhythm. Brain Res 439:383-387. CrossRef Medline

Pelli DG (1997) The VideoToolbox software for visual psychophysics: transforming numbers into movies. Spat Vis 10:437-442. CrossRef Medline

Richardson JTE (2011) Eta squared and partial eta squared as measures of effect size in educational research. Educ Res Rev 6:135-147. CrossRef

Rizzuto DS, Madsen JR, Bromfield EB, Schulze-Bonhage A, Kahana MJ (2006) Human neocortical oscillations exhibit theta phase differences between encoding and retrieval. Neuroimage 31:1352-1358. CrossRef Medline

Rutishauser U, Ross IB, Mamelak AN, Schuman EM (2010) Human memory strength is predicted by theta-frequency phase-locking of single neurons. Nature 464:903-907. CrossRef Medline
Saupe K, Schröger E, Andersen SK, Müller MM (2009) Neural mechanisms of intermodal sustained selective attention with concurrently presented auditory and visual stimuli. Front Hum Neurosci 3:58. CrossRef Medline

Schott BH, Wüstenberg T, Wimber M, Fenker DB, Zierhut KC, Seidenbecher CI, Heinze HJ, Walter H, Düzel E, Richardson-Klavehn A (2013) The relationship between level of processing and hippocampal-cortical functional connectivity during episodic memory formation in humans. Hum Brain Mapp 34:407-424. CrossRef Medline

Scoville WB, Milner B (1957) Loss of recent memory after bilateral hippocampal lesions. J Neurol Neurosurg Psychiatry 20:11-21. CrossRef Medline

Senkowski D, Schneider TR, Foxe JJ, Engel AK (2008) Crossmodal binding through neural coherence: implications for multisensory processing. Trends Neurosci 31:401-409. CrossRef Medline

Song S, Miller KD, Abbott LF (2000) Competitive hebbian learning through spike-timing-dependent synaptic plasticity. Nat Neurosci 3:919-926. CrossRef Medline

Staresina BP, Davachi L (2009) Mind the gap: binding experiences across space and time in the human hippocampus. Neuron 63:267-276. CrossRef Medline

Staudigl T, Hanslmayr S (2013) Theta oscillations at encoding mediate the context-dependent nature of human episodic memory. Curr Biol 23: 1101-1106. CrossRef Medline

Summerfield C, Mangels JA (2005) Coherent theta-band EEG activity predicts item-context binding during encoding. Neuroimage 24:692-703. CrossRef Medline

Summerfield C, Greene M, Wager T, Egner T, Hirsch J, Mangels J (2006) Neocortical connectivity during episodic memory formation. PLoS Biol 4:e128. CrossRef Medline

Tadel F, Baillet S, Mosher JC, Pantazis D, Leahy RM (2011) Brainstorm: a user-friendly application for MEG/EEG analysis. Comput Intell Neurosci 2011:879716. CrossRef Medline

Tulving E (2002) Episodic memory: from mind to brain. Annu Rev Psychol 53:1-25. CrossRef Medline

Van Veen BD, van Drongelen W, Yuchtman M, Suzuki A (1997) Localization of brain electrical activity via linearly constrained minimum variance spatial filtering. IEEE Trans Biomed Eng 44:867-880. CrossRef Medline 